\title{
CONTROLES POR SELEÇÃO E REJEIÇÃO EM TREINOS DE DISCRIMINAÇÃO CONDICIONAL E TESTES DE EQUIVALENCIA ${ }^{1}$
}

\author{
SELECT AND REJECT CONTROLS IN CONDITIONAL DISCRIMINATION \\ TRAINING AND EQUIVALENCE TESTS
}

\author{
William Ferreira Perez e Gerson Yukio Tomanari
}

UNIVERSIDADE DE SÃO PAULO, BRASIL

\begin{abstract}
RESUMO
Falhas na formação de classes de estímulos equivalentes têm sido explicadas, ocasionalmente, em função dos controles de estímulo (ou topografias de controle de estímulo) que ocorrem ao longo dos treinos de discriminaçáo condicional e nos testes de equivalência. $\mathrm{O}$ presente artigo pretende apresentar, didaticamente, as implicaçóes de dois controles de estímulo particulares, o de escolha por seleção do estímulo programado como S+ e o de rejeição daquele programado como $S$ - sobre a formação de classes de estímulos equivalentes em tarefas de matching-to-sample (MTS) envolvendo dois estímulos de comparação. Para tanto, é apresentada, após uma breve revisão da literatura de equivalência, a análise teórica de Carrigan e Sidman (1992), bem como resultados experimentais que a dão suporte. Por fim, dados de pesquisas recentes são discutidos frente a possíveis propostas de investigação que isolariam experimentalmente o estabelecimento de diferentes controles de estímulo.

Palavras-chave: topografias de controle de estímulo, controle por seleção, controle por rejeição, equivalência de estímulos, matching to sample.
\end{abstract}

\begin{abstract}
Failures in equivalence class formation have been explained, occasionally, as a function of stimulus controls (or stimulus control topographies) that occur during the conditional discrimination training and in the equivalence tests. The present article aims to present, didactically, the implications of two particular stimulus controls, the selection of the programmed $S+$ and the rejection of the $S$ - to the formation of equivalence classes in matching-to-sample tasks involving two comparison stimuli. Thus, it is presented, after a brief review of the equivalence literature, the theoretical analysis by Carrigan and Sidman (1992) and the experimental results that support it directly. Finally, recent data are discussed in regard to possible future researches that would isolate the establishment of different stimulus controls.
\end{abstract}

Key words: stimulus control topographies, select control, reject control, stimulus equivalence, matching to sample.

O presente artigo pretende apresentar, didaticamente, as implicações de diferentes controles de estímulos (ou topografias de controle de estímulo), em particular a seleção do estímulo programado como $\mathrm{S}+$ e a rejeição daquele programado como S-, sobre a formação de classes de estímulos equivalentes em tarefas de matching to sample (MTS, escolha de acordo com o modelo) envolvendo dois estímulos de comparação. Para tanto, serão apresentados, após uma breve revisão da literatura de equivalência, a análise teórica realizada por Carrigan

\footnotetext{
${ }^{1}$ O presente trabalho é parte da dissertação de mestrado do primeiro autor e contou com o apoio da Fundação de Amparo à Pesquisa do Estado de São Paulo (FAPESP) por meio de uma bolsa de Mestrado (Processo no 06/05165-4). A versão eletrônica da dissertação (Perez, 2008), intitulada "Movimentos dos olhos e topografias de controle de estímulos em treino de discriminação condicional e testes de equivalência", pode ser encontrada em www.teses.usp.br. O primeiro autor é bolsista de doutorado da FAPESP (Processo n ${ }^{\circ}$ 09/51176-6). O segundo autor é pesquisador bolsista do CNPq. Os autores agradecem as contribuições dos professores Deisy das Graças de Souza e Júlio C. de Rose (UFSCar), membros da banca de qualificação e defesa da dissertação. Contato para correspondência: William Ferreira Perez, Rua Pascoal Vita, 405, apto. 81, Vila Madalena, São Paulo-SP, 05445-000, Brasil. E-mail: willperę@ymail.com.
} 
e Sidman (1992) e os resultados experimentais que dão suporte direto a tal análise (Johnson \& Sidman, 1993; Magnusson, 2002; Perez, 2008). Por fim, serão levantadas e discutidas algumas questões referentes às implicações do controle por seleção e do controle por rejeição sobre a formação de classes de equivalência bem como a possibilidade de pesquisas futuras que isolariam experimentalmente o estabelecimento de ambos controles de estímulo.

\section{Comportamento Operante e \\ Controle de Estímulos}

Ao emitirem respostas, os organismos modificam seu ambiente. Tais modificações (conseqüências) alteram a probabilidade de ocorrência futura do responder. Respostas seguidas de conseqüências reforçadoras têm a sua probabilidade de ocorrência aumentada. Respostas seguidas de conseqüências punitivas têm sua probabilidade de ocorrência reduzida. Os estímulos que antecedem a ocasião em que uma dada resposta é seguida de suas conseqüências, por sua vez, também adquirem controle sobre o responder, aumentando ou diminuindo a sua probabilidade de ocorrência a depender da função exercida pela conseqüência da resposta, reforçadora ou punitiva (Skinner, 1953).

Quando o responder ocorre na presença de um dado estímulo e não de outro, ele se encontra sob controle discriminativo. Por exemplo: em uma tarefa no computador, dois estímulos visuais são apresentados na tela, B1 e B2. Escolher B1 é seguido do acúmulo de pontos em um contador; escolher B2, não. Caso o participante escolha (clicando com o mouse, por exemplo) o estímulo B1 e não o B2, consistentemente, diz-se que o participante apresenta um responder discriminado. O estímulo na presença do qual respostas de escolha são reforçadas (pontos), no caso B1, é chamado de $\mathrm{S}^{\mathrm{D}}$ (estímulo discriminativo) ou
$\mathrm{S}^{+}$. Alternativamente, o estímulo na presença do qual respostas não são reforçadas, no caso, B2, é chamado de $S^{\Delta}$ ou $S$. Este é um exemplo típico de um procedimento de discriminação simples.

O responder discriminado pode, ainda, ocorrer sob controle de outro estímulo, de tal forma que B1 e B2 podem ou não ocasionar a emissão da resposta, dependendo da presença de um estímulo condicional (A1 ou A2). Sendo assim, escolher B1, e não B2, só é reforçado na presença (é condicional à presença) de A1, enquanto que escolher B2, e não B1, só é reforçado na presença (é condicional à presença) de A2. Tem-se aqui um caso de discriminação condicional.

\section{EquivalêNCIA de Estímulos}

O procedimento de MTS tem sido tradicionalmente utilizado no estudo de discriminações condicionais (Sidman, 1994). Neste procedimento, a cada tentativa, um modelo é apresentado simultaneamente a dois ou mais comparações de tal forma que os estímulos comparação têm sua função discriminativa $\left(S^{\mathrm{D}}\right.$ ou $\mathrm{S}^{\Delta}$ ) alterada de acordo com o estímulo modelo presente (Catania, 1998/1999; Cumming \& Berryman, 1965). A discriminação condicional treinada é usualmente representada pela junção de duas letras maiúsculas que correspondem aos conjuntos de estímulos envolvidos no treino. Considerando o exemplo do parágrafo anterior, $\mathrm{AB}$ corresponderia ao treino da discriminação condicional entre os estímulos do conjunto $\mathrm{A}$ e os estímulos do conjunto B, por exemplo. A primeira letra do par representa os estímulos modelo, enquanto a segunda representa os estímulos comparação. Nesse caso, os estímulos do conjunto A seriam sucessivamente apresentados como modelo e os estímulos do conjunto B seriam simultaneamente apresentados como comparações. Os estímulos de cada conjunto, por sua vez, são representados pela letra que 
corresponde ao conjunto seguida de um dígito (A1, A2, An, como estímulos do conjunto A; B1, B2, Bn, como estímulos do conjunto B) (ver de Rose, 1993, 1996).

No exemplo adiante, considerar-se-á um treino de MTS envolvendo três conjuntos (A, B e C) formados por dois estímulos cada, em uma seqüência de tentativas na qual, em cada uma delas, um modelo é apresentado simultaneamente a dois estímulos comparação. Considerando um treino $\mathrm{AB}$ e $\mathrm{BC}$, tem-se como resultado as seguintes relações entre os estímulos de cada conjunto: A1B1 e A2B2 para o treino $\mathrm{AB}$; $\mathrm{B} 1 \mathrm{C} 1$ e $\mathrm{B} 2 \mathrm{C} 2$, para o treino $\mathrm{BC}$ - de tal forma que o primeiro estímulo da relação é sempre o estímulo modelo e o segundo é sempre o comparação. Tomando como exemplo o treino $\mathrm{AB}$, segue uma descrição dos procedimentos necessários ao estabelecimento das relações condicionais (A1B1 e A2B2) entre os estímulos desses conjuntos: um dado estímulo é apresentado como modelo (A1 ou A2) seguido, usualmente depois de uma resposta de observação (tocar o estímulo, exemplo), da apresentação de dois estímulos comparação (B1 e B2) dos quais somente um pode ser escolhido. $\mathrm{Na}$ presença de A1, escolher B1 é seguido de reforço; escolher B2, não. Na presença de A2, escolher B2 é seguido de reforço; escolher B1, não. Já para o treino $\mathrm{BC}$, para o qual corresponderia o treino das relações B1C1 e B2C2: na presença de B1, escolher C1 é seguido de reforço; escolher C2, não. Na presença de B2, escolher C2 é seguido de reforço; escolher C1, não (Sidman, 1994).

Relações condicionais, ensinadas como descrito acima, podem gerar relações de equivalência entre os estímulos que as compõem. Nesse caso, convencionalmente, diz-se que os estímulos desses três conjuntos (A, B e C) formam uma classe de estímulos equivalentes caso eles se mostrem substituíveis entre si no controle do comportamento. De acordo com a teoria dos conjuntos, elementos podem ser equivalentes se apresentarem, em relação a si mesmos ou aos demais, as seguintes propriedades: reflexividade, simetria e transitividade. A verificação dessas propriedades é efetuada por meio de testes que aferem a emergência de relações condicionais que não foram ensinadas diretamente no treino (AB/ $\mathrm{BC})$ : as relações $\mathrm{AA}, \mathrm{BB}$ e $\mathrm{CC}$, no caso da reflexividade; $\mathrm{BA}$ e $\mathrm{CB}$, no caso da simetria; e AC, no caso da transitividade (Sidman, 1994; Sidman \& Tailby, 1982).

Para que a reflexividade seja demonstrada, cada estímulo deve apresentar, com relação a si próprio, o mesmo tipo de relação condicional treinada em relação aos estímulos dos outros conjuntos. Para tanto, o mesmo estímulo é apresentado tanto como modelo quanto como comparação. Se durante o treino $\mathrm{AB}$ os participantes aprenderam a escolher o estímulo comparação B1 diante do modelo A1 e B2 diante de A2, por exemplo, a reflexividade (AA) seria demonstrada se, tendo A1 e A2 como modelos e comparações, A1 fosse escolhido diante de A1 e A2 diante de A2. O mesmo se aplica aos estímulos dos demais conjuntos (escolher B1 diante de B1; C1 diante de C1 e assim por diante).

Para que a simetria seja demonstrada, estímulos modelos e comparação devem ter suas funções permutadas de tal forma que B1 e B2, apresentados como comparações durante o treino $\mathrm{AB}$, passam a ser apresentados como modelos seguidos de A1 e A2 como comparações, por exemplo. Caso o participante escolha A1 diante de B1 e A2 diante de B2, a emergência da relação simétrica BA será demonstrada. O mesmo se dá para a relação $\mathrm{CB}$.

A transitividade (AC), por sua vez, é verificada quando o participante passa a responder relacionando modelos e comparações que nun- 
ca foram apresentados em um mesmo treino, mas que, durante essa fase, foram relacionados a outros estímulos em comum (tanto A quanto $\mathrm{C}$, durante o treino, foram relacionados com B). Ou seja, depois de aprender as relações entre os estímulos dos conjuntos AB e BC, o participante é capaz de escolher o estímulo comparação C1 diante do modelo A1 e C2 diante de A2.

Por fim, existe ainda o teste de equivalência ou, em outras palavras, simetria da transitividade (CA). Para que essa propriedade seja demonstrada, o participante deve ser capaz de escolher A1 diante de C1 e A2 diante de C2 (de Rose, 1993, 1996; Sidman, 1994; Sidman \& Tailby, 1982).

As ocorrências de treinos de discriminação condicional bem-sucedidos seguidos de falhas nos testes de formação de classes de estímulos equivalentes (reflexividade, simetria, transitividade e equivalência) indicam a possibilidade de que outras variáveis, além de um bom desempenho no treino, estejam envolvidas no estabelecimento de relações de equivalência (cf. de Rose, 1996; de Rose, Kato, Thé, \& Kledaras, 1997; McIlvane, Serna, Dube, \& Stromer, 2000). Dentre essas variáveis estão as possíveis variações no controle de estímulo que se dão ao longo do treino de relações e condicionais e testes de equivalência, as também chamadas "topografias de controle de estímulo" (TCE) (Dube \& McIlvane, 1996; Goulart, Mendonça, Barros, Galvão, \& McIlvane, 2005; McIlvane, 1998; McIlvane \& Dube, 1992; McIlvane \& Dube, 2003; McIlvane et al., 2000; Ray, 1969; Serna, Lionello-DeNolf, Barros, Dube, \& McIlvane, 2004), que determinam o responder do participante na tarefa.

\section{Matching to Sample e Controles por Seleção e Rejeição}

Carrigan e Sidman (1992) apontam algo curioso acerca do controle de estímulos que se estabelece durante os treinos de MTS e que, por sua vez, pode ser responsável por falhas nos testes de equivalência. Segundo esses autores, a descrição de uma relação condicional como uma relação entre o estímulo modelo e o estímulo comparação escolhido pode desconsiderar uma variável importante: os diferentes controles de estímulo que podem afetar as escolhas do participante, os quais podem ser compreendidos como sendo diferentes "topografias de controle de estímulo (TCE)".

O termo TCE se refere às características físicas, relações estruturais e propriedades controladoras dos estímulos. Uma TCE é um paralelo conceitual de topografia de respostas: da mesma maneira que topografia de resposta distingue entre várias formas de resposta que produzem o mesmo resultado mensurado, TCE distingue várias formas de relações de controle de estímulos que produzem a mesma medida de desempenho (itálico acrescido pelo autor) (Serna et al., 2004, p. 255).

Tal como citado acima, Carrigan e Sidman (1992) apontam que, em uma tarefa de MTS com dois estímulos comparação, uma mesma escolha pode ser controlada por diferentes TCEs. De acordo com esses autores, uma relação condicional é formada por dois componentes: a) o par de estímulos que se relaciona condicionalmente (modelo-comparação) e b) o "tipo de relação de controle" que se

\footnotetext{
${ }^{2}$ Embora Carrigan e Sidman (1992) tenham utilizado os termos type control (tipo de controle), type relation (tipo de relação) e type of comparion-stimulus control (tipo de controle de estímulo pelo comparação), o presente trabalho utilizará o termo "topografia de controle de estímulo (TCE)" (Dube \& McIlvane, 1996; Goulart et al., 2005; McIlvane, 1998; McIlvane \& Dube, 1992; McIlvane \& Dube, 2003; McIlvane et al., 2000; Ray, 1969; Serna et al., 2004) para se referir à mesma classe de eventos comportamentais. Quando o controle for especificado, os termos "controle por seleção" e "controle por rejeição" serão utilizados.
} 
estabelece (seleção ou rejeição) e que define a maneira como o participante responde na presença desse par - o que foi chamado pelos autores de componente comportamental. Em uma discriminação condicional como a considerada anteriormente $(\mathrm{AB})$, um estímulo modelo A1 é apresentado simultaneamente a dois comparações, B1 e B2, na tela de um computador. Clicar com o mouse sobre B1 é sempre reforçado; clicar sobre B2 não. Como mostra

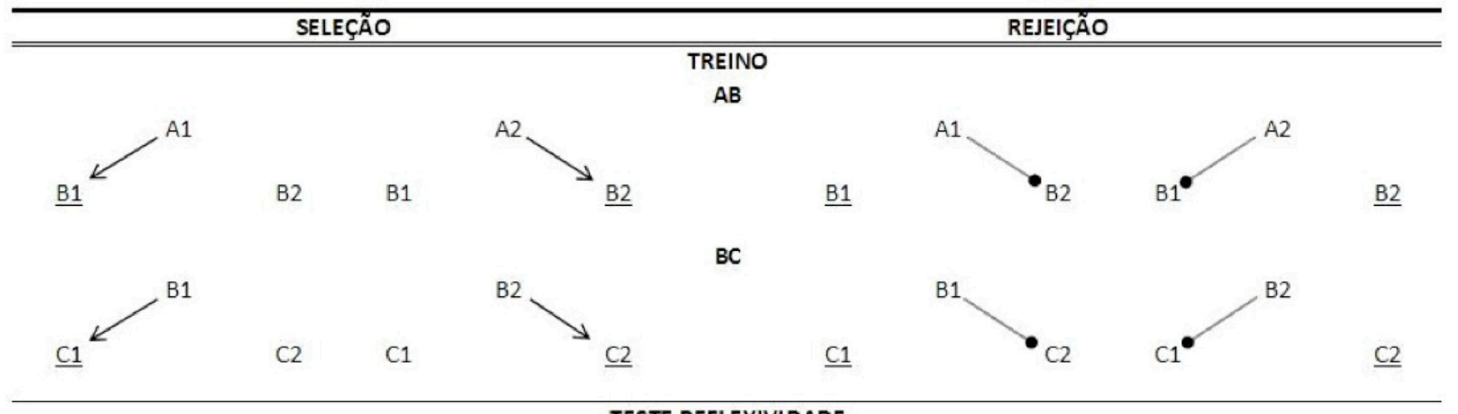

TESTE REFLEXIVIDADE

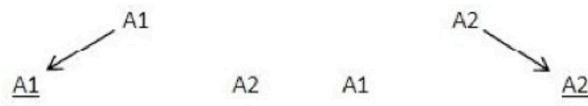

AA
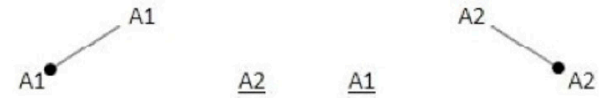

BB<smiles>Br[Mg]Br</smiles>

B2

B1
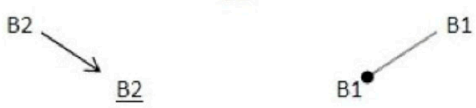

A2

$\underline{\mathrm{A} 1}$

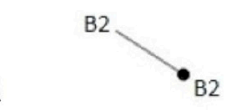

CC
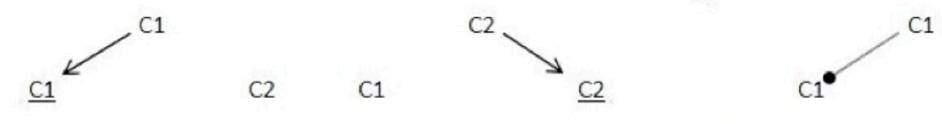

$\underline{B 2}$

$\underline{B 1}$

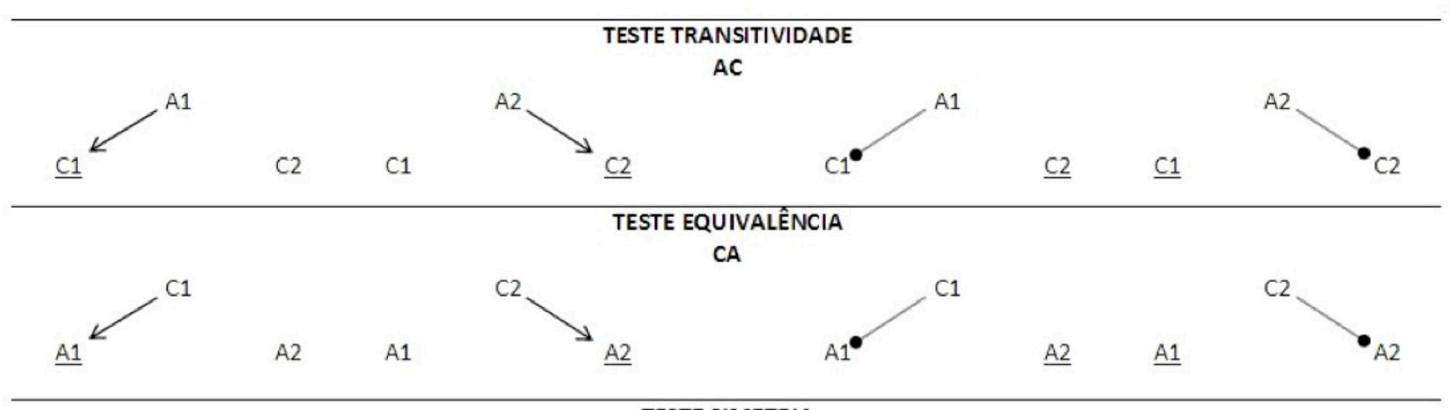

\section{TESTE SIMETRIA}

BA

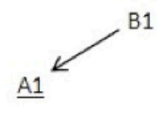

A. 2

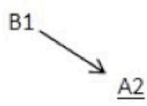

A1
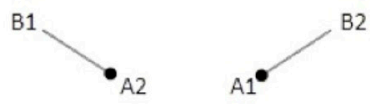

B2

CB

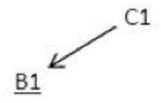

B2

B1

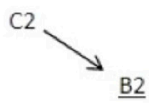

B1
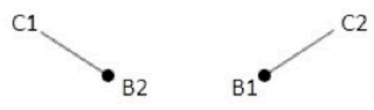

B2

Figura 1. Cada triângulo de estímulos corresponde a uma tentativa de MTS. O estímulo do vértice superior é apresentado como modelo; os estímulos dos vértices inferiores esquerdo e direito são apresentados como comparações, sendo que o estímulo sublinhado corresponde àquele escolhido. As linhas indicam o par de estímulos que controla o responder. Quando a ponta da linha é uma seta, indica controle por seleção, quando é um circulo, indica controle por rejeição (cf. de Rose et al., 2000). 
a parte superior da Figura 1, embora a resposta registrada pelo computador seja sempre a mesma, caso o participante escolha sempre o estímulo programado como "correto" (S+), existe pelo menos duas TCEs possíveis para a mesma resposta registrada (clicar sobre B1). Quando a resposta de escolha é controlada pelo par de estímulos modelo-S+, diz-se que o controle é por seleção. Nesse caso, diz-se A1 controla a seleção de B1 (clicar em B1). Quando a resposta de escolha é controlada pelo par de estímulos modelo-S-, diz-se que o controle é por rejeição; nesse caso, A1 controla a rejeição de B2, levando o participante a escolher outro estímulo disponível (clicar em B1). Em ambos os casos, embora as TCEs sejam distintas, a resposta de escolha é a mesma. Existe a possibilidade, ainda, de que ambas TCEs vigorarem simultaneamente. Nesse caso, a escolha de B1 é controlada tanto pela seleção de B1 (S+) quando pela rejeição de B2 (S-).

Cabe ressaltar que, embora inúmeras TCEs possam ser estabelecidas durante um treino no procedimento MTS, aqui, somente o controle por seleção e por rejeição serão considerados.

Controle por Seleção, Controle por ReJEIÇÃO E SUAS IMPLICAÇÕES PARA A FormaÇÃo de Classes de Estímulos Equivalentes: A Hipótese de Carrigan e Sidman (I992)

Durante o treino de discriminação condicional na tarefa de MTS tal como tem sido tradicionalmente utilizada (Sidman, 1994), pouco pode ser dito a-cerca das TCEs (em particular dos controles por seleção e rejeição) envolvidas na escolha do estímulo comparação. Para tanto, faz-se necessário realizar, posteriormente a um desempenho adequado nos treinos, testes que demonstrem a TCE em operação (Arantes, 2008; Carr, Wilkinson, Blackman, \& McIlvane, 2000; Carrigan \& Sidman, 1992; Cumming \& Berryman, 1965; de Rose, Hidalgo, \& Vasconcelos, 2000; Dixon \& Dixon, 1978; Goulart et al., 2005; Grisante, 2007; Johnson \& Sidman, 1993; Kato, de Rose, \& Faleiros, 2008; Magnusson, 2002; McIlvane, Kledaras, Munson, King, de Rose, \& Stoddard, 1987; McIlvane, Withstandley, \& Stoddard, 1984; Perez, 2008; Stromer \& Osborne, 1982; Vasconcellos, 2003). Carrigan e Sidman (1992), em uma análise teórica, propuseram que alguns dos testes das propriedades que definem uma classe de estímulos equivalentes (reflexividade, transitividade e equivalência) seriam possíveis indicadores das TCEs envolvidas nos treinos de discriminação condicional.

Segundo Carrigan e Sidman (1992), para diferentes TCEs seriam esperados diferentes resultados nos testes de algumas das propriedades da equivalência. Para os testes de reflexividade, transitividade e equivalência com número de nodos ímpar ${ }^{3}$, resultados opostos seriam observados a depender da TCE estabelecida. Como mostra a Tabela 1, quando o controle por seleção é estabelecido ao longo de todas as tentativas apresentadas durante o treino, resultados positivos nos testes serão esperados. Por outro lado, quando o controle por rejeição é estabelecido, os resultados devem ser negativos. Nos testes de simetria, os resultados serão os mesmos independentemente da TCE em vigor.

\footnotetext{
3 O termo "nodo" corresponde ao conjunto de estímulos com os quais dois ou mais outros conjuntos se relacionam. Por exemplo, em um treino AB/BC, existe apenas um conjunto de estímulo que é relacionado a dois outros conjuntos. $\mathrm{O}$ conjunto $\mathrm{B}$, nesse caso, corresponde ao nodo das relações $\mathrm{AB} / \mathrm{BC}$. Supondo um treino $\mathrm{AB} / \mathrm{BC} / \mathrm{CD}$, têm-se, por sua vez, dois nodos: $\mathrm{B}$, que se relaciona com os conjuntos $\mathrm{A}$ e $\mathrm{C}$; e $\mathrm{C}$ que se relaciona com os conjuntos $\mathrm{B}$ e D. O termo nodo também se aplica aos estímulos do conjunto (Fields \& Verhave, 1987; Fields, Adam, Verhave, \& Newman, 1990; Fields, Landon-Jimenez, Buffington, \& Adams, 1995).
} 
Tabela 1

Resultados esperados nos testes de transitividade, simetria, equivalência e reflexividade com número de nodos ímpar, a depender da TCE estabelecida (controle por seleção ou por rejeição) ao longo do treino de discriminação condicional. O símbolo " $\checkmark$ " indica resultados positivos. O símbolo “-" indica resultados negativos.

\begin{tabular}{ccccc}
\hline & Transitividade & Simetria & Equivalência & Reflexividade \\
\hline Seleção & $\checkmark$ & $\checkmark$ & $\checkmark$ & $\checkmark$ \\
& & & & \\
Rejeição & - & $\checkmark$ & - & - \\
\hline
\end{tabular}

A Figura 1 apresenta uma análise caso a caso dos resultados esperados nos testes de reflexividade, transitividade, equivalência e simetria a depender da TCE estabelecida durante o treino. Supõe-se um treino AB/BC (com um único nodo, B).

Inicialmente, será considerado o caso no qual, durante o treino, o controle por seleção é estabelecido para todas as tentativas apresentadas. Por exemplo, quando os pares A1-B1 e B1-C1 controlam o responder por seleção, ou seja, quando diante de A1 o participante seleciona B1 (escolhe B1) e diante de B1 seleciona C1 (escolhe C1) - o mesmo se dará para os demais pares modelo-S+ (por exemplo, A2-B2, B2-C2). Nos testes de reflexividade, o participante deverá apresentar, na relação de cada estímulo consigo mesmo, a mesma TCE estabelecida durante o treino. Assim, quando o par A1-A1 for apresentado, o participante deverá selecionar A1 diante de A1 (escolherá A1). O mesmo se dará para os demais pares de estímulo An-An, Bn-Bn, Cn-Cn. Nos testes de transitividade (AC), em conformidade com as relações treinadas, quando o par A1-C1 ${ }^{4}$ for apresentado, diante de A1 o participante deverá selecionar C1 (escolherá C1) - e diante de An, deverá selecionar Cn (escolherá Cn). Algo similar se dará nos testes de equivalência (CA), ou seja, quando o par C1-A1 for apresentado, o participante deverá diante de C1 selecionar A1 (escolherá A1) - e diante de Cn selecionar An (escolherá An).

Resultados opostos aos descritos acima serão observados caso o controle por rejeição seja estabelecido para todas as tentativas de treino. Por exemplo, quando os pares A1-B2 e B2-C1 controlam o responder por rejeição, ou seja, quando diante de A1 o participante rejeita B2 (e escolhe o outro estímulo apresentado, B1) e diante de B1 rejeita C2 (ou seja, escolhe C1) - o mesmo se dará para os demais pares modelo-S- (por exemplo, A2-B1 e B1-C2). Nos testes de reflexividade, em conformidade com o treino realizado, o participante deverá, diante de A1 rejeitar A1, ou seja, escolherá A2, e não A1. O mesmo ocorrerá para os demais pares de estímulo An-An, Bn-Bn, Cn-Cn. Nos testes de transitividade, de acordo com o treino realizado, quando o par A1-C1 for apresentado, diante de A1 o participante deverá rejeitar $\mathrm{C} 1$, ou seja, escolherá C2. Nos testes de equivalência, de modo similar, quando o par C1A1 for apresentado, o participante deverá diante de C1 rejeitar A1, ou seja, escolherá A2. Algo similar se dará caso, durante o treino, os pares A2B1 e B1-C2 controlarem o responder por rejeição.

A Figura 2 mostra um exemplo das diferentes respostas de escolha esperadas nos testes de transitividade, a depender da TCE estabelecida durante o treino. De acordo com as áreas circuladas, para diferentes TCEs, exis-

\footnotetext{
${ }^{4}$ O par de estímulos A1 (modelo) - C1 (comparação) é apresentado em uma tentativa na qual A1 é modelo e C1 e C2 são comparações, correspondente ao teste de transitividade (AC). Em todos os exemplos, deve-se considerar que o par de estímulos que controla o responder (e.g., An-Cn) é apresentado junto de outro comparação (e.g., Cx).
} 


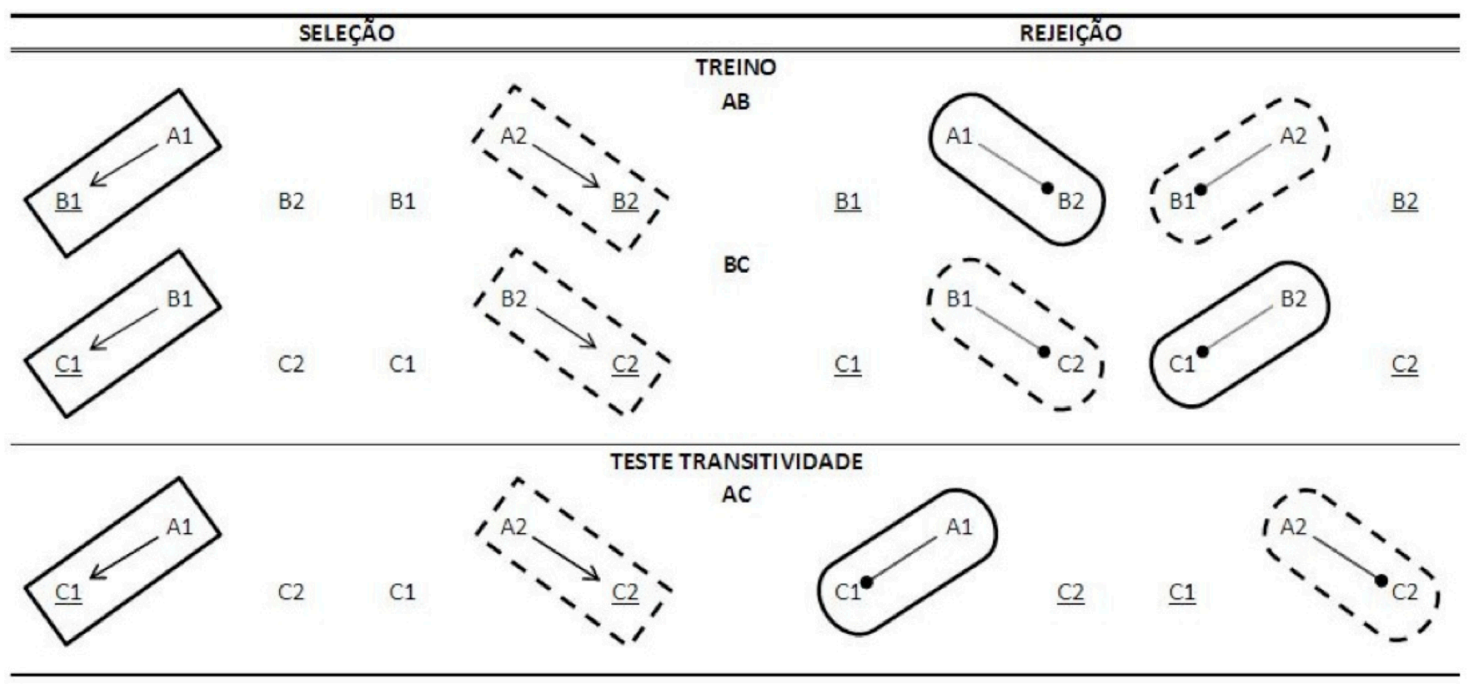

Figura 2. Supondo um treino AB/BC, são indicadas as diferentes "rotas de emergência" nos testes de transitividade AC, a depender da TCE estabelecida. O termo "rota de emergência”, aqui, é utilizado como um artifício didático para diferenciar graficamente os efeitos do controle por seleção e por rejeição sobre o desempenho no treino e nos testes.

tem diferentes "rotas de emergência" de novas relações entre estímulos durante os testes. O termo "rota de emergência", aqui, é utilizado como um artifício didático para diferenciar graficamente os efeitos do controle por seleção e por rejeição sobre o desempenho nos treinos e nos testes.

Resta pontuar, ainda, que as diferenças observadas nos resultados de transitividade e equivalência são moduladas pelo número de nodos que separa os conjuntos de estímulos apresentados nos testes. Até aqui, foram considerados testes (AC e CA) nos quais os conjuntos de estímulos apresentados, durante o treino $(\mathrm{AB} / \mathrm{BC})$, eram separados por um único nodo (B). No entanto, como mostra a Figura 3, quando o número de nodos que separa os estímulos apresentados nos testes é par, os mesmos resultados são esperados independentemente da TCE estabelecida durante o treino. Por exemplo, em um treino AB/BC/CD seguido dos testes $\mathrm{AD}$ e DA. Supondo que os pares A1-B1, B1-C1 e C1-D1 controlem o responder por seleção, no testes de transitividade, quando o par A1-D1 for apresentado, diante de A1 o participante deverá selecionar D1 (escolherá D1). No teste de equivalência, quando o par D1-A1 for apresentado, diante de D1 o participante deverá selecionar A1 (escolherá A1). Agora, supondo que os pares A1-B2, B2-C1 e C1-D2 controlem o responder por rejeição, no teste de transitividade, quando o par A1-D2 for apresentado, o participante deverá diante de A1 rejeitar D2, ou seja, também escolherá D1. No caso da equivalência, supondo que os pares A2-B1, B1-C2 e C2-D1 controlem o responder por rejeição, quando o par D1-A2 for apresentado no teste, o participante deverá diante de D1 rejeitar A2, ou seja, também escolherá A1.

Em suma, de acordo com Carrigan e Sidman (1992), são previstos diferentes resultados para os testes de transitividade e equivalência somente quando o número de nodos dos conjuntos de estímulos envolvidos nos testes for impar. Considerando um treino AB/BC/CD/ $\mathrm{DE}$, poder-se-á prever que, dadas diferentes TCEs, diferentes resultados serão esperados nos seguintes testes de transitividade e equivalência: AC e CA (somente um nodo: B), BD e DB (somente um nodo: C), CE e EC (somente um nodo: D) e, por fim, AE e EA (três nodos: B, C e D). Quando o número de nodos for par, 


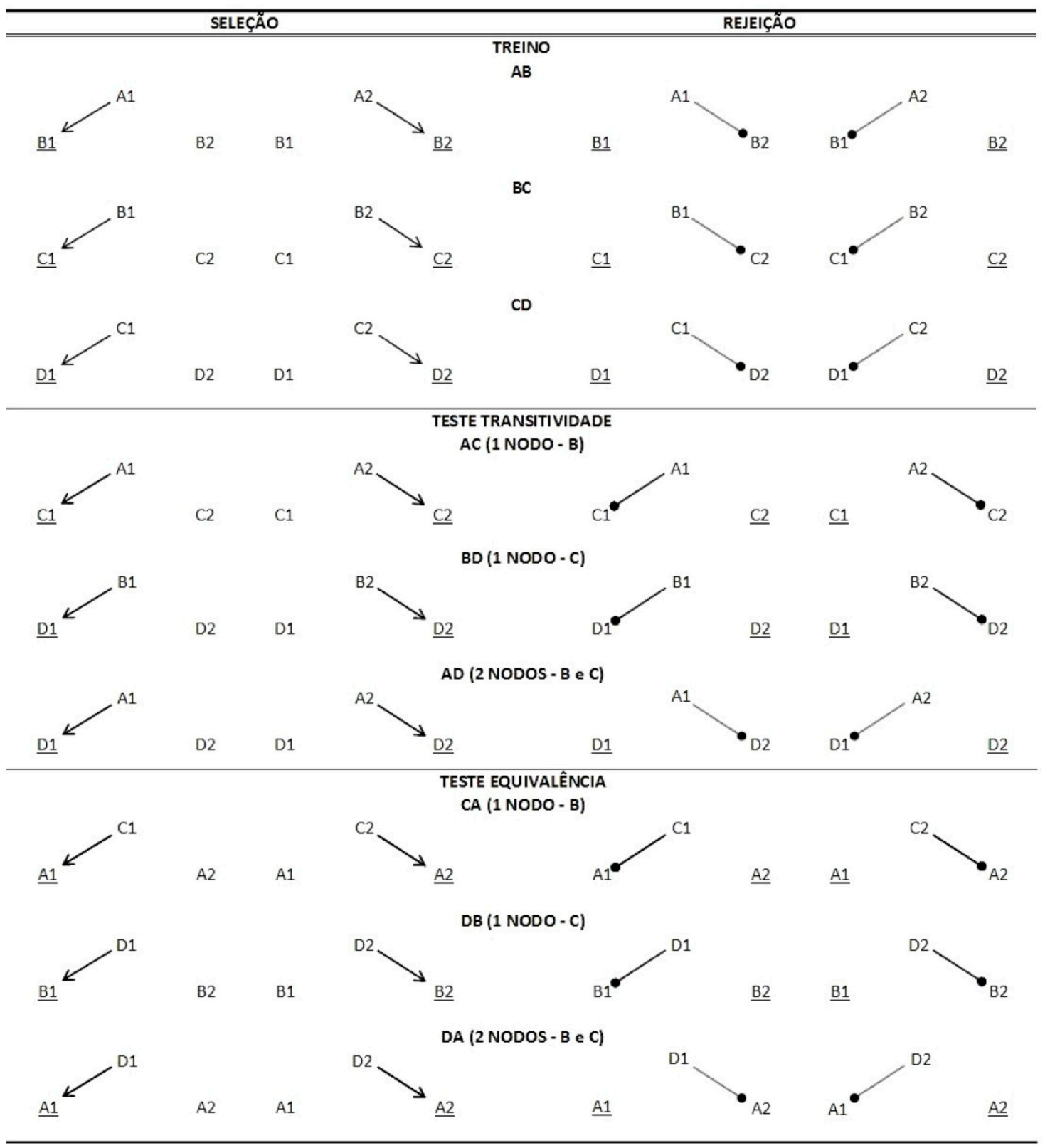

Figura 3. Dado um treino $\mathrm{AB} / \mathrm{BC} / \mathrm{CD}$, são apresentados os diferentes estímulos escolhidos durante os testes de transitividade e equivalência a depender da TCE estabelecida e, também, do número de nodos.

apenas os testes de reflexividade podem apresentar diferentes resultados já que os estímulos apresentados são sempre do mesmo conjunto.

Nos testes de simetria (BA e CB), ao contrário dos testes de reflexividade, transitividade e equivalência, os mesmos resultados (estímulos escolhidos) seriam esperados in- dependentemente da TCE estabelecida (ver Figura 1). No caso de um treino em que o controle por seleção tenha sido estabelecido (por exemplo, no qual o par de estímulos A1-B1 controla o responder por seleção), no teste de simetria BA, o participante deverá, na presença de B1 selecionar A1 (escolherá A1). 
No caso de um treino em que o controle por rejeição tenha sido estabelecido (por exemplo, no qual o par de estímulos A2-B1 controla o responder por rejeição), nos testes de simetria BA, o participante deverá, na presença de B1 rejeitar A2, ou seja, escolherá A1. Em ambos os casos descritos, independentemente da TCE que se estabeleça, o mesmo comparação será escolhido pelo participante (no exemplo acima, A1). Algo similar se dará nos testes de simetria $\mathrm{CB}$, quando os pares $\mathrm{B} 1-\mathrm{C} 1$ controlarem o responder por seleção e B2-C1 controlarem o responder por rejeição, por exemplo. Em ambos os casos, B1 será escolhido.

Resta ressaltar, ainda, que as previsões de resultados encontradas nas análises de Carrigan e Sidman (1992) partem do pressuposto de que o controle, seja por seleção ou por rejeição, deve ocorrer de forma constante e majoritária ao longo das tentativas de treino. Quando ambas as TCEs ocorrem simultaneamente para uma mesma tentativa ou quando em algumas tentativas ocorre o controle por seleção e em outras o controle por rejeição, os resultados nos testes não acompanharão as análises apresentadas até aqui.

\section{INVESTIGAÇÓES EXPERIMENTAIS SOBRE A} Hipótese de Carrigan e Sidman (i 992): Consideraçóes sobre os Procedimentos

Antes de descrever os estudos que investigaram empiricamente as hipóteses levantadas por Carrigan e Sidman (1992), faz-se necessário apresentar mais detalhadamente os procedimentos utilizados para favorecer a variável independente comum a esses estudos: a manipulação da TCE estabelecida durante o treino. Embora exista uma diversidade de procedimento utilizados para favorecer o estabelecimento dos controles por seleção e rejeição (e.g. Arantes, 2008; de Rose et al., 2000; Goulart et al., 2005; Hamasaki, 2009; McIlvane,
Kledaras, Callahan, \& Dube, 2002; McIlvane et al., 1987; Vasconcellos, 2003), aqui, serão descritos apenas os procedimentos utilizados nos estudos a serem apresentados na seqüência.

Nos três experimentos que serão descritos adiante, de modo a favorecer o estabelecimento do controle por rejeição, durante o treino, foram utilizadas diferentes proporções de $\mathrm{S}+$ e S-, como sugerido por Carrigan e Sidman (1992). Tal procedimento consiste em aumentar a proporção de $\mathrm{S}+$ ou S- apresentados a depender da TCE que se pretende produzir. Considerando um treino (AB, por exemplo) no qual um modelo (A1) é apresentado simultaneamente a dois comparações, um S+ (B1) e outro S- (B2), ao longo das tentativas, novos $\mathrm{S}+(\mathrm{W} 1, \mathrm{~W} 2, \mathrm{~W} 3)$ ou S- (X1, X2, X3) são apresentados a depender da TCE que se pretende instalar. Quando o controle por seleção é visado, o S+ (B1) é sempre apresentado e, a cada tentativa, um dos quatro S- (B2, X1, X2, X3) é apresentado simultaneamente a ele; ou seja, a cada tentativa, o S+ é apresentado junto de um S- diferente. Quando o controle por rejeição é visado, o S- (B2) é sempre apresentado e, a cada tentativa, um dos quatro $\mathrm{S}+(\mathrm{B} 1, \mathrm{~W} 1, \mathrm{~W} 2, \mathrm{~W} 3)$ é apresentado simultaneamente a ele; ou seja, a cada tentativa, o S- é apresentado junto de um $\mathrm{S}+$ diferente.

Em dois dos estudos descritos (Johnson \& Sidman, 1993; Magnusson, 2002), além de uma maior proporção de S+ ou S-, foi utilizado também um procedimento adicional de "dica atrasada". Nesse procedimento, para favorecer a rejeição do S-, o S+ desaparecia da tela e era substituído por um quadrado branco que podia ser escolhido como comparação. Respostas ao quadrado branco eram, a partir de então, seguidas das conseqüências programadas para os $\mathrm{S}+$. Inicialmente, tal dica era apresentada 0,1 segundo depois da apresentação dos comparações. O período necessário à apresentação da dica foi progressivamente aumentado com 
o decorrer do treino. De forma análoga, para favorecer o controle por seleção, transcorrido um período na ausência de respostas do participante, o comparação S- desaparecia da tela e somente o S+ ficava disponível para escolha.

Outra característica comum a esses experimentos é o número de comparações utilizados. Em todos eles, a cada tentativa, um estímulo modelo era apresentado no centro da tela simultaneamente a dois outros comparações cuja posição variava randomicamente entre os quatro cantos do monitor. O uso de dois comparações torna a seleção e a rejeição equiprováveis (um S+ versus um S-). Além disso, no caso da rejeição, na medida em que o estímulo que controla a resposta de escolha não é o estímulo escolhido, o uso de três ou mais comparações dificulta a análise de qual foi o S- rejeitado.

\section{Os Estudos Experimentais}

De acordo com Carrigan e Sidman (1992), os primeiros dados experimentais que corroboram a hipótese de que o controle por rejeição seria seguido por resultados negativos nos testes de reflexividade, transitividade e equivalência com número de nodos ímpares podem ser encontrados em Carrigan (1986). No entanto, os primeiros dados publicados em periódico científico foram os de Johnson e Sidman (1993), apresentados preliminarmente em congresso por Johnson e Sidman (1991).

No estudo de Johnson e Sidman (1993), três adultos foram submetidos ao treino das relações AB e BC, separadamente. Depois de aprendidas, tais relações eram apresentadas juntas em uma mesma sessão, $\mathrm{AB} / \mathrm{BC}$. Ao longo das tentativas de treino (linha de base), foi utilizada uma maior proporção de $\mathrm{S}+$ e a dica atrasada favorecendo rejeição do S- (retirada do $\mathrm{S}+$ ). Uma vez atingido o critério de aprendizagem, na seqüência, foram realizados testes de simetria (BA e $\mathrm{CB})$, transitivi- dade (AC) e equivalência (CA). Depois dos testes para a linha de base $\mathrm{AB} / \mathrm{BC}$, foram ensinadas as relações $\mathrm{CD}$. Uma vez atingido o critério em um treino com todas as relações $\mathrm{AB} / \mathrm{BC} / \mathrm{CD}$, foram realizados testes de simetria $(\mathrm{BA}, \mathrm{CB}$ e DC), transitividade (AC, BD e AD) e equivalência (CA, DB e DA). Para um dos participantes, foi realizado o teste de reflexividade (AA, BB, CC e DD).

Os resultados observados por Johnson e Sidman (1993) confirmam as predições de Carrigan e Sidman (1992). De modo geral, os participantes apresentaram porcentagens de acerto próximas de zero (fracassaram) nos testes de transitividade (AC e BD), equivalência (CA e BD) e reflexividade (AA, BB, CC e DD), cujos conjuntos de estímulos eram relacionados entre si a partir de um único nodo. Os autores discutem que o estabelecimento do controle por rejeição levou à formação de classes de estímulos equivalentes (A1B2C1D2 e A2B1C2D1) distintas daquelas esperadas a partir do treino realizado (A1B1C1D1 e A2B2C2D2). Nos testes de simetria, transitividade e equivalência, cujos conjuntos de estímulos eram relacionados a partir de dois nodos, os participantes obtiveram alta porcentagem de acerto. Isso corrobora a previsão de Carrigan e Sidman, de que o número de nodos seria um parâmetro crítico no uso dos testes de equivalência para aferir os efeitos dos diferentes controles de estímulos estabelecidos durante o treino.

Seguindo Johnson e Sidman (1993), pelo menos dois outros estudos, ainda não publicados, fornecem suporte direto a hipótese de Carrigan e Sidman (1992): Magnusson (2002) e Perez (2008). Embora tais estudos tenham trazido contribuições metodológicas para a investigação das TCEs por meio da medida dos movimentos dos olhos dos participantes, os dados aqui apresentados fazem referência somente ao desempenho na tarefa de MTS.

Magnusson (2002 ou, na forma resumida, Magnusson, 2003), em duas fases experimentais 
distintas (Rejeição e Seleção), para diferentes conjuntos de estímulos, favoreceu o estabelecimento de diferentes TCEs: na fase de Rejeição (RJ), o controle por rejeição; na fase de Seleção (SL), o controle por seleção. Para o participante $\mathrm{KAA}$, a ordem das condições experimentais foi RJ à SL; para o participante YXN, a ordem foi invertida, SL à RJ.

$\mathrm{Na}$ Fase RJ, foi realizado um treino AB/ $\mathrm{BC}$ e o controle por rejeição foi favorecido por meio dos mesmos procedimentos de Johnson e Sidman (1993). Primeiramente, foi realizado o treino das relações $A B$. Uma vez atingido o critério, foram treinadas, separadamente, as relações BC. Em seguida, foi realizado o treino do conjunto $\mathrm{AB} / \mathrm{BC}$. Caso apresentasse um responder estável em uma sessão com tentativas de linha de base $(\mathrm{AB} / \mathrm{BC})$ sem conseqüências diferenciais para acerto e erro, o participante era submetido aos testes de transitividade (AC), simetria (BA e $\mathrm{CB}$ ), equivalência (CA) e reflexividade ( $\mathrm{AA}, \mathrm{BB}, \mathrm{CC})$, nessa seqüência.

$\mathrm{Na}$ Fase SL, foi realizado um treino DE/ EF e o controle por seleção foi favorecido utilizando-se uma maior proporção de S- e dica atrasada favorecendo a seleção do S+ (retirada do S-). Primeiramente, foi realizado o treino das relações DE. Uma vez atingido o critério, foram treinadas, separadamente, as relações EF. Em seguida, foi realizado o treino do conjunto DE/ EF. Caso apresentasse um responder estável em uma sessão com tentativas de linha de base (DE/EF) sem conseqüencias diferenciais para acerto e erro, o participante era submetido aos testes de transitividade (EF), simetria (ED e FE), equivalência (FE) e reflexividade (DD, EE e FF).

Os resultados observados por Magnusson (2002) durante os testes replicaram aqueles apresentados por Johnson e Sidman (1993), confirmando as predições de Carrigan e Sidman (1992). Na Fase RJ, o participante KAA apresentou porcentagem de acerto igual ou próxima a zero nos testes de reflexividade (AA, $\mathrm{BB}$ e CC), transitividade (AC) e equivalência (CA). YXN apresentou resultados similares, porém não tão evidentes. $\mathrm{O}$ fracasso nos testes de reflexividade só foram apresentadas depois de repetidos os testes e os dados do teste de transitividade (AC) ficaram ao nível do acaso com relação a porcentagem de acertos. $\mathrm{Na}$ Fase SL, os participantes apresentaram alta porcentagem de acertos em todos os testes realizados. Os resultados da Fase SL sugerem que o estabelecimento do controle por seleção é condição necessária para a formação da classe de equivalência planejada pelo experimentador.

Perez (2008) replicou o estudo de Magnusson (2002) implementando algumas modificações. Uma delas foi a inserção de uma fase inicial de linha de base na qual nenhuma TCE era favorecida. Além disso, nas demais fases, as TCEs foram favorecidas sem o uso da dica atrasada, utilizando o método da manipulação das proporções de S+ ou S-. Os demais parâmetros do estudo foram mantidos idênticos aos de Magnusson (2002). Quatro participantes universitários foram submetidos inicialmente à fase de Linha de Base (LB). Na seqüencia, dois deles foram submetidos às demais fases (Rejeição e Seleção) na seqüência RJ à SL (P1 e P2) e dois na seqüência SL à RJ (P3 e P4). Durante a Fase LB, todos os participantes apresentaram alta porcentagem de acertos em todos os testes realizados. Na Fase RJ, somente P1 apresentou desempenho em acordo com as previsões de Carrigan e Sidman (1992). Nos testes, esta participante falhou em apresentar as relações de transitividade (DF), equivalência (FD) e reflexividade (DD, EE e FF). P1 apresentou um único acerto $(6 \%)$ nas sessões de transitividade (DF), equivalência (FD) e em um dos testes de reflexividade EE. Nas sessões de reflexividade DD e FF, a participante não emitiu respostas de escolha corretas. Nos testes de simetria, P1 apresentou alta porcentagem de acerto. P2, na Fase 
RJ, levou o dobro de sessões de treino quando comparada a P1. Esse dado favorece a hipótese de que a participante aprendeu a selecionar cada um dos S+ ao invés de rejeitar o S-. Isso explicaria os resultados positivos durante os testes. Os dados de P3 e P4 parecem sugerir o estabelecimento de um responder condicional generalizado. $\mathrm{Na}$ Fase SL, todos os participantes apresentaram altas porcentagens de acerto durante os testes.

No estudo de Perez (2008), embora os dados de P1 na Fase RJ, em contraposição às Fases LB e SL, corroborem fortemente a hipótese de Carrigan e Sidman (1992), a falha no estabelecimento do controle por rejeição para os demais participantes parece apontar que a fase de linha de base e/ou a não utilização do procedimento de dica atrasada podem comprometer o estabelecimento do responder por rejeição. $\mathrm{O}$ autor discute a necessidade de se investigar procedimentos capazes de isolar o estabelecimento dessas TCEs de modo a verificar seus efeitos sobre a formação de classes de equivalência.

\section{Consideraçóes Finais}

Já que para diferentes TCEs (controle por seleção ou por rejeição) são previstos diferentes resultados no que tange a formação de diferentes classes, as análises de Carrigan e Sidman (1992), aliadas aos dados obtidos por estudos experimentais (Johnson \& Sidman, 1993; Magnusson, 2002; Perez, 2008), dão suporte para que o estudo das TCEs envolvidas em treinos de discriminação condicional seja tomado como variável crítica para o entendimento formação de classes de estímulos equivalentes.

Embora existam evidências de que o controle por rejeição seja responsável por falhas na formação das classes de estímulos equivalentes esperadas pelo experimentador (Johnson \& Sidman, 1993; Magnusson, 2002; Perez, 2008) e alguns pesquisadores sugiram que o controle exclusivo por seleção permita a equivalência (cf., Carrigan \& Sidman, 1992 - descrito acima), os resultados de outros estudos apontam que ainda não estão claras as implicações dos controles por seleção e rejeição sobre a formação de classes de equivalência. Existem evidências, por exemplo, de que a formação de classes de equivalência pode ocorrer em condições em que ambas TCEs são simultaneamente estabelecidas (Arantes, 2008; Carr et al., 2000; de Rose et al., 2000; Hamasaki, 2009; Kato et al., 2008; Vasconcellos 2003) e que, em alguns casos (Arantes, 2008; Carr et al., 2000), isso poderia favorecer a emergência das relações durante os testes (Arantes, 2008; Carr et al., 2000). No estudo de Arantes (2008), por exemplo, nas fases em que uma máscara encobriu o S- na maior parte das tentativas, favorecendo o controle exclusivo por seleção, não foi observada a formação de classes. O mesmo ocorreu na fase em que a máscara encobriu o $\mathrm{S}+$ na maior parte das tentativas, favorecendo o controle exclusivo por rejeição. A formação de classes só foi observada nas fases em que a máscara encobriu o $\mathrm{S}+$ em metade das tentativas e o S- na outra metade. Nesse caso, ambos controles tiveram o seu estabelecimento favorecido. No estudo de Carr et al. (2000), realizado com participantes que apresentavam desenvolvimento verbal mínimo, quatro dos cinco indivíduos que apresentaram resultados positivos nos testes de equivalência também apresentaram desempenhos que sugerem controle por seleção e por rejeição durante a realização de testes nos quais o $\mathrm{S}+$ ou o $\mathrm{S}$ - eram substituídos por estímulos novos. Os estudos de Arantes (2008) e Carr et al. (2000) levantam a hipótese de que tanto o controle por seleção quanto por rejeição talvez sejam necessários à formação de classes de estímulos equivalentes. Tais resultados, no entanto, não são conclusivos na medida em que carecem de generalidade e 
controles adicionais. Ainda assim, em conjunto com outros esperimentos (Vohnsin \& Sidman, 1993; Magnusson, 2002: Prez, 2008), sustentam a importância de se investigar o papel do controle por seleção e por rejeição na formação de classes de equivalência e abrem novas questões como, por exemplo: em que medida a formação de classes pode ser favorecida quando ambas TCEs estão presentes? O controle isolado por seleção seria de fato suficiente? As classes formadas por rejeição também seriam classes de equivalência funcional? O que ocorreria quando essas TCEs "competem" pela emergência das relações testadas?

Os argumentos apresentados acima apontam a necessidade de se investigar o efeito dos controles exclusivos por seleção e rejeição sobre a formação de classes de estímulos equivalentes. No entanto, procedimentos capazes de garantir o estabelecimento do controle por seleção ou rejeição ainda precisam ser mais bem desenvolvidos e investigados (Goulart et al., 2005; Perez, 2008). Goulart et al. (2005) sugere que estudos futuros deveriam buscar promover “(...) o desenvolvimento de procedimentos mais efetivos de modelagem de controle de estímulo para o estudo do efeito de diferentes TCEs nos comportamentos emergentes nos testes de equivalência de estímulos” (p. 301, traduzido pelo autor). Ainda, se estudos posteriores pudessem se dedicar, não à avaliação das TCEs estabelecidas durante o treino a despeito das contingências programadas pelo experimentador, mas sim ao controle experimental direto dessas TCEs, poder-se-ia favorecer uma maior coerência entre as TCEs planejadas pelo experimentador e estabelecidas no responder do participante (cf. Goulart et al., 2005; ver também Perez, 2008).

Alguns estudos recentes têm se dedicado a essa tarefa, desenvolvendo novos procedimentos capazes de produzir e isolar o controle por seleção e por rejeição. Hamasaki (2009), por exemplo, desenvolveu um procedimento que consiste em encobrir todos os estímulos (modelo e comparações) com uma janela que pode ser aberta pelo participante por meio do clique do mouse sobre um botão localizado abaixo da área de apresentação do estímulo. Um clique sobre esse botão (resposta de observação) produz a abertura da janela permitindo que o estímulo seja observado por um breve período de tempo. Em uma tarefa de MTS, Hamasaki favoreceu o estabelecimento dos controles por seleção e/ou rejeição manipulando a resposta de observação por meio de duas estratégias distintas. Em uma delas, para favorecer a seleção, na maioria das tentativas, o participante era impedido de observar o S-; para favorecer a rejeição, o participante era impedido de observar o $\mathrm{S}+$. Na outra, as probabilidades de que as respostas de observação dessem acesso ao $\mathrm{S}+$ ou ao S- foram manipuladas sistematicamente. Em ambas, a identificação dos controles por seleção e rejeição ocorria ao longo do treino de discriminação condicional, fato que possibilita monitorá-los e, eventualmente, alterá-los previamente aos testes de equivalência. Nessa linha de investigação, portanto, estudos futuros deverão desenvolver as potencialidades desse procedimento. Entre outras possibilidades, seria importante, para fins experimentais, isolar os controles do responder por seleção e rejeição. Uma alternativa para isso seria, por exemplo, não apenas impedir a observação de um dos estímulos, mas também garantir a observação exclusiva do S+ e do S-.

\section{REFERÊNCIAS}

Arantes, A. K. (2008). Pré-requisitos para aprendizagem relacional em crianças com histórico de fracasso escolar. Dissertação de mestrado, Programa de Pósgraduação em Educação Especial, Universidade 
Federal de São Carlos, São Carlos.

Carr, D., Wilkinson, K. M., Blackman, D., \& McIlvane,

W. J. (2000). Equivalence classes in individuals with minimal verbal repertoires. Journal of the Experimental Analysis of Behavior, 74, 101-114.

Carrigan, P. F. (1986). Conditional discrimination and transitive relations: A theoretical and experimental analysis. Unpublished doctoral dissertation, Northeastern University, Boston.

Carrigan, P. F., \& Sidman, M. (1992). Conditional discrimination and equivalence relations: A theoretical analysis of control by negative stimuli. Journal of the Experimental Analysis of Behavior, 58, 193-204.

Catania, A. C. (1999). Aprendizagem: comportamento, linguagem e cognição. Porto Alegre, RS: Artes Médicas do Sul. (Originalmente publicado em 1998).

Cumming, W. W., \& Berryman, R. (1965). The complex discriminated operant: Studies of matching to sample and related problems. Em D. I. Mostofski (Orgs.), Stimulus generalization (pp. 284-329). Stanford, CA: Stanford University Press.

de Rose, J. C. (1993). Classes de estímulos: implicações para uma análise comportamental da cognição. Psicologia: Teoria e Pesquisa, 9, 283-303.

de Rose, J. C. (1996). Controlling factors in conditional discriminations and tests of equivalence. Em T. R. Zentall \& P. M. Smeets (Orgs.), Stimulus class formation in bumans and animals (pp. 253-277). North Holland, Amsterdam, NL: Elsevier.

de Rose, J. C., Hidalgo, M., \& Vasconcelos, M. (2000). Are sample S+ controlling relations necessary and sufficient for the formation of equivalence classes? Manuscrito não publicado.

de Rose, J. C., Kato, O. M., Thé, A. P., \& Kledaras, J. B. (1997). Variáveis que afetam a formação de classes de estímulos: estudos sobre efeitos do arranjo de treino. Acta Comportamentalia, 5, 143-163.

Dixon, M. H., \& Dixon, L. S. (1978). The nature of standard control in children's matching-tosample. Journal of the Experimental Analysis of Behavior, 30, 205-212.
Dube, W. V., \& McIlvane, W. J. (1996). Some implications of a stimulus control topography analysis of emergent behavior and stimulus classes. Em T. R. Zental \& P. M. Smeets (Orgs.), Stimulus Class Formation in Humans and Animals (pp. 197-218). North Holland, Amsterdam, NL: Elsevier.

Fields, L., Adam, B. J., Verhave, T., \& Newman, S. (1990). The effects of nodality on the formation of equivalence classes. Journal of the Experimental Analysis of Behavior, 53, 345-358.

Fields, L., Landon-Jimenez, D. V., Buffington, D. M., \& Adams, B. J. (1995). Maintained nodaldistance effects in equivalence classes. Journal of the Experimental Analysis of Behavior, 64, 129-145.

Fields, L., \& Verhave, T. (1987). The strutucture of equivalence classes. Journal of the Experimental Analysis of Behavior, 48, 317-332.

Goulart, P. R. K., Mendonça, M. B., Barros, R. S., Galvão, O. F., \& McIlvane, W. J. (2005). A note on select- and reject-controlling relations in the simple discrimination of capuchin monkeys (Cebus apella). Behavioural Processes, 69, 295-302.

Grisante, P. C. (2007). Consistência entre formação de classes de equivalência e topografias de controle de estímulo estabelecidas no treino de discriminações condicionais. Dissertação de mestrado, Programa de Pósgraduação em Educação Especial, Universidade Federal de São Carlos, São Carlos.

Hamasaki, E. (2009). Respostas de observação na tarefa de pareamento ao modelo: analisando topografias de controle de estimulos e seus efeitos sobre a formacão de equivalência. Tese de doutorado, Programa de Pós-graduação Psicologia Experimental, Universidade de São Paulo, São Paulo. Johnson, C., \& Sidman, M. (1991, May). Stimulus classes established by sample-and-S- conditional-discrimination performance. Paper presented at the meeting of the Association for Behavior Analysis, Atlanta, Georgia. Johnson, C., \& Sidman, M. (1993). Conditional discrimination and equivalence relations: control by negative stimuli. Journal of the Experimental Analysis of Behavior, 59, 333-347.

Kato, O. M., de Rose, J. C., \& Faleiros, P. B. (2008). 
Topography of responses in conditional discrimination influences formation of equivalence classes. The Psychological Record, 58, 245-267.

Magnusson, A. (2002). Topography of eye movements under select and reject control. Dissertação de mestrado, Shriver Center, Northeastern University, Boston. Magnusson, A. (2003). Topography of eye movements under select and reject control. Experimental Analysis of Human Behavior Bulletin, 21, 42.

McIlvane, W. J. (1998). Teoria da coerência da topografia de controle de estímulos: uma breve introdução. Temas em Psicologia, 6, 185-189.

Mcllvane, W. J., \& Dube, W. V. (1992). Stimulus control shaping and stimulus control topographies. The Behavior Analyst, 15, 89-94.

McIlvane, W. J., \& Dube, W. V. (2003). Stimulus control topography coherence theory: Foundations and extensions. The Behavior Analyst, 26, 195-213.

McIlvane, W. J., Kledaras, J. B., Callahan, T. C., \& Dube, W. V. (2002). High probability stimulus control topographies with delayed $\mathrm{S}+$ onset in a simultaneous discrimination procedure. Journal of the Experimental Analysis of Behavior, 77, 189-198.

McIlvane, W. J., Kledaras, J. B., Munson, L. C., King, K. A., de Rose, J. C., \& Stoddard, L. T. (1987). Controlling relations in conditional discrimination and matching by exclusion. Journal of the Experimental Analysis of Behavior, 48, 187-208.

McIlvane, W. J., Serna, R., Dube, W. V., \& Stromer, R. (2000). Stimulus control topography coherence and stimulus equivalence: Reconciling test outcomes with theory. Em J. Leslie \& D. E. Blackman (Orgs.), Issues in experimental and applied analysis of human behavior (pp. 85-101). Reno: Context Press.

McIlvane, W. J., Withstandley, J. K., \& Stoddard, L. T. (1984). Positive and negative stimulus relations in severely retarded individuals' conditional discrimination. Analysis and Intervention in
Developmental Disabilities, 4, 235-251.

Perez, W. F. (2008). Movimentos dos olhos e topografias de controle de estímulos em treino de discriminação condicional e testes de equivalência. Dissertação de mestrado, Programa de Pós-graduação Psicologia Experimental, Universidade de São Paulo, São Paulo.

Ray, B. A. (1969). Selective attention: The effects of combining stimuli which control incompatible behavior. Journal of the Experimental Analysis of Behavior, 12, 539-550.

Serna, R. W., Lionello-DeNolf, K. M., Barros, R. S., Dube, W. V., \& Mcllvane, W. J. (2004). Teoria de coerência de topografias de controle de estímulos na aprendizagem discriminativa: da pesquisa básica à aplicação. Em M. M. Hübner \& M. Marinotti (Orgs.), Análise do comportamento para a educação: contribuição recentes (pp. 253-284). Campinas: ESETec.

Sidman, M. (1994). Equivalence relations and behavior: A research history. Boston: Authors Cooperative.

Sidman, M., \& Tailby, W. (1982). Conditional discrimination vs. matching to sample: An expansion of the testing paradigm. Journal of the Experimental Analysis of Behavior, 37, 5-22.

Skinner, B. F. (1953). Science and human behavior. New York: The Free Press.

Stromer, R., \& Osborne, J. G. (1982). Control by adolescent's arbitrary matching-to-sample by positive and negative stimulus relations. Journal of the Experimental Analysis of Behavior, 37, 329-348.

Vasconcellos, M. (2003). Aprendizagem relacional em crianças com baixo desempenho escolar. Dissertação de mestrado, Programa de Pós-graduação em Educação Especial, Universidade Federal de São Carlos, São Carlos.

Artigo submetido em 15 de junho de 2009 Aceito em 9 de novembro de 2009

Publicação referente ao $2^{\circ}$. Semestre de 2008, impressa em maio de 2011 